\title{
QPSK Modulation and Demodulation System Using SIW Interconnect
}

\author{
Ning Wang ${ }^{\mathrm{a}}$, Xiao-Chun Li, Wei-Xin, Xiao-Jian Ma, Yan Shao, and Jun-Fa \\ Mao
}

Key Lab of Ministry of Education for Research of Design and EMC of High Speed Electronic Systems,

Shanghai Jiao Tong University, Shanghai, China

aningw@sjtu.edu.cn

Keywords: Quadrature Phase Shift Keying (QPSK); substrate integrated waveguide (SIW); modulation and demodulation; pseudo-random binary sequences (PRBS)

\begin{abstract}
In this paper, design and fabrication of a substrate integrated waveguide (SIW) with 14$28 \mathrm{GHz}$ bandwidth is implemented. The SIW-based system with Quadrature Phase Shift Keying (QPSK) modulation and demodulation technique is designed and implemented. The measurement results show that the proposed system has up to 8 Gbps transmission data rate, which doubles the transmission rate compared with traditional mixing system. The demonstrated system is verified to be an effective interconnect system for ultra high speed transmission.
\end{abstract}

\section{Introduction}

A substrate integrated waveguide (SIW), presenting a planar and integrated form with metalized hole arrays instead of metallic sidewalls of the rectangle waveguide, has been widely used for the design of a new class of millimeter-wave components [1]. Due to easy fabrication process and a relatively low cost of production [2]-[3], SIW interconnects are often employed as high speed data transfer physical channels in the field of high frequency communication system. With the highpass characteristics of SIW, baseband signals must be modulated before entering the SIW interconnect and demodulated after extracting from the same interconnect channel to satisfy high speed data transmission. A waveguide system yielding 5 Gbps data transmission capacity is designed and validated by using mixing technique experimentally where excellent signal integrity characteristics are obtained [4]. However, traditional mixing, whose channel bandwidth usage is not very efficient due to its double sideband modulation, is no longer prevalent for high speed and large bandwidth signal transmission. To further increase the channel capacity, Quadrature Phase Shift Keying (QPSK) and 16 Quadrature Amplitude Modulation (16QAM) can be employed. In this paper, an experimental prototype of SIW covering wide bandwidth is presented. Based on QPSK scheme, data communication utilizing SIW interconnect in high-speed signaling applications is addressed. At last experimental platform is established and verified for the proposed communication system.

\section{System Implementation}

\section{Design and Fabrication of SIW.}

The center frequency of SIW is $21 \mathrm{GHz}$ with $14 \mathrm{GHz}$ cut-off frequency, thus the usable bandwidth spans from $14 \mathrm{GHz}$ up to about $28 \mathrm{GHz}$, i.e. about $14 \mathrm{GHz}$. As we know, microstrip line is widely used in the traditional coplanar integrated circuits whose characteristic impedance is set to $50 \Omega$ in common practice. In order to realize the integration between microstrip line and SIW, an efficient taper transition structure with good impedance matching is designed and optimized here. The detailed dimensions and design parameters of the SIW structure are summarized in Table 1. According to these design rules, the final SIW structure is shown in Fig. 1 (a). 
table 1. dimensions of siw, microstrip line and tapered transition (unit: mm)

\begin{tabular}{|c|c|c|c|c|}
\hline / SIW & Microstrip & $\begin{array}{c}\text { Tapered } \\
\text { transition }\end{array}$ & $\begin{array}{c}\text { Via } \\
\text { spacing } \\
\text { distance }\end{array}$ \\
\hline Width & 7.016 & 1 & 3.776 & 0.8 \\
\hline Length & 36 & 3.7 & 6.4 & $\begin{array}{c}\text { Via } \\
\text { diameter }\end{array}$ \\
\hline Height & 0.035 & 0.035 & 0.035 & 0.4 \\
\hline
\end{tabular}

Fullwave simulations are employed to illustrate the performance of SIW with a Rogers 5880 substrate and the results are shown in Fig. 1 (b). It can be seen that the return loss is better than 15 $\mathrm{dB}$ while the insertion loss is less than $0.5 \mathrm{~dB}$ in the entire designed bandwidth except for the cutoff frequency nearby. Since the microstrip line in Fig. 1 (a) lies in the middle of substrate, odd modes will be activated on the one side of SIW rather than even modes due to their heterodromous distributed electric field in transverse section. Furthermore, there is no apparent coupling between the dominant mode TE10 and other higher order modes in the wide bandwidth to support lager data rate transmission in SIW. To validate the performance of designed SIW, two SIW prototypes with different test fixtures composed by metallic $\mathrm{Cu}$ and $\mathrm{Al}$ are fabricated for comparison as shown in Fig. 2.

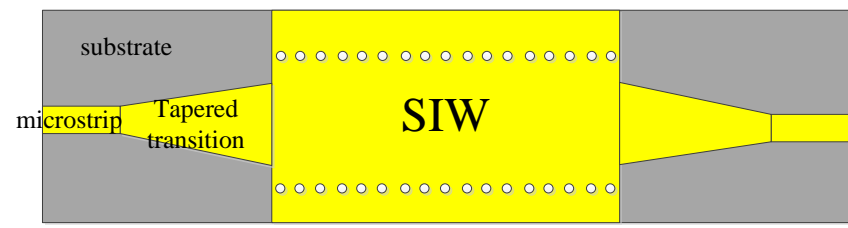

(a)

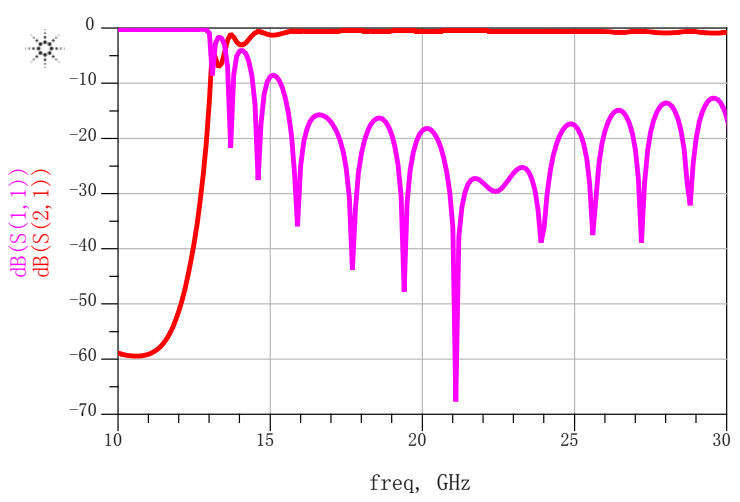

(b)

Fig. 1. Designed structure and simulated S-parameter of SIW. (a) Top view of SIW; (b) return loss and insertion loss.

\section{QPSK Transmission System.}

In QPSK, each 2-bit symbol is mapped to a cosine carrier phase shifted with multiples of $\pi / 2$ [5]. Four different phase shifts have to be realized in order to achieve a QPSK scheme. Thus, whether 900 phase shift is been fulfilled becomes a key issue that decides the system performance directly.

The overall QPSK transmission system based on SIW is shown in Fig. 3. In the modulation part, baseband signal is splitted into two data flows with half of original data rate. And then, the detached data 


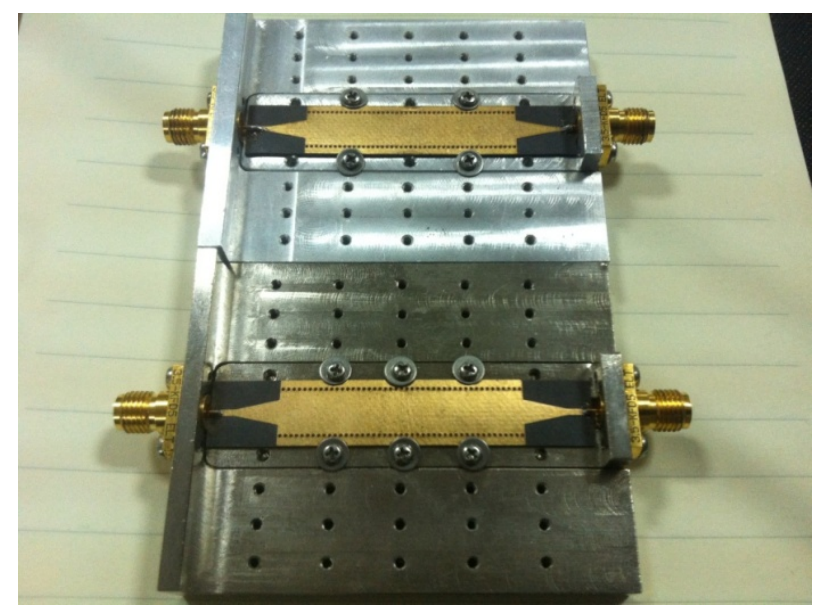

Fig. 2. Fabricated prototypes.

experience up conversion by mixers with orthorhombic local carriers respectively. It is necessary to note that the frequency band near the cutoff frequency of SIW should be avoided when the baseband data is transferred to bandpass channel on mixer. In echo demodulation process, modulation signal is separated into two parts by power divider, and each is carried out down conversion process with two orthogonal carriers by mixing again. In order to compensate the conversion loss due to mixers during modulation and demodulation, an extra amplifier is added after SIW. Finally, the data signals are connected with two low pass filters to remove ultraharmonics from image mixing.

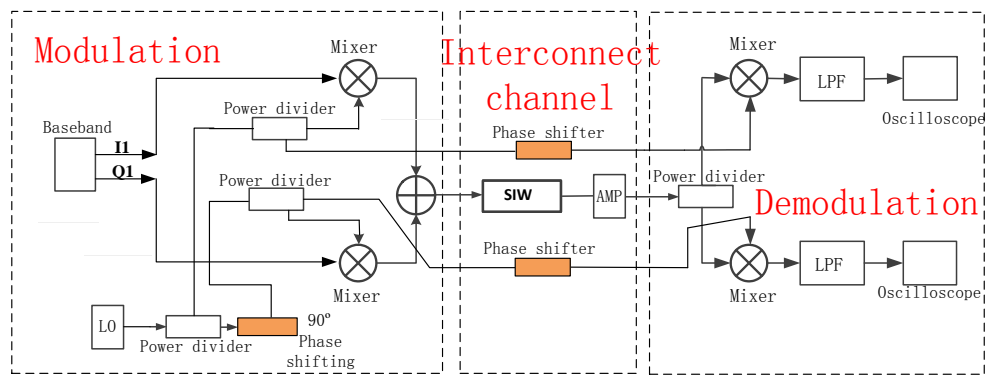

Fig. 3. Architecture of QPSK transmission system.

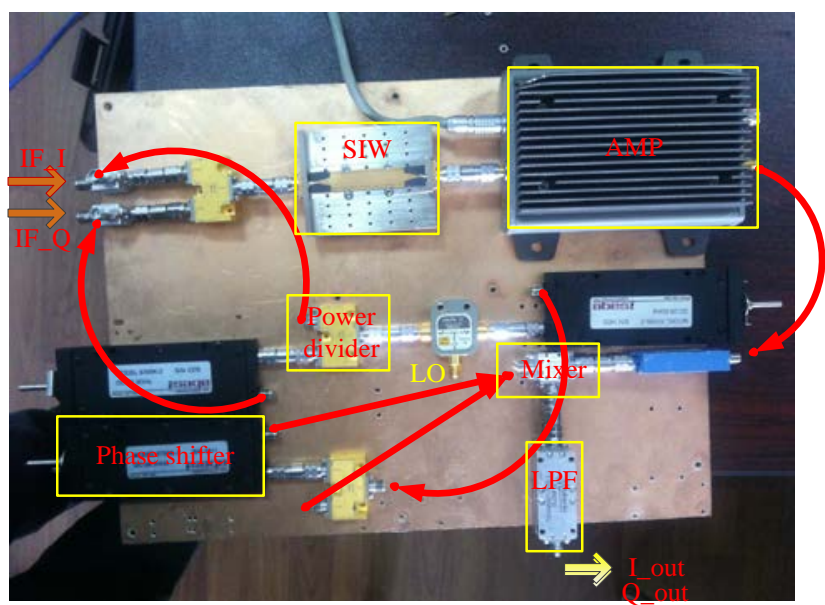

Fig. 4. System prototype. 


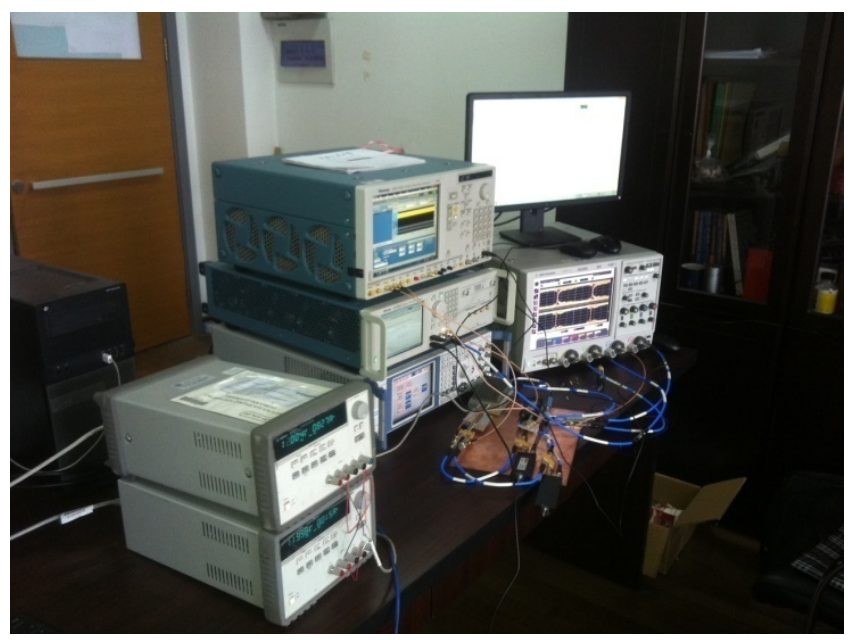

Fig. 5. Experimental environment.

It is worth mentioning that three duplicated phase shifters are employed in this system, one of which are used to come out $90^{\circ}$ phase shift and the other two serve to compensate phase shift caused by SIW, AMP and high frequency cables in SIW channel.

\section{Results and Discussion}

The system prototype and test environment are shown in Fig. 4 and Fig. 5, respectively. The separate baseband signals in channel I and Q are two independent rectangle pseudo-random binary sequences (PRBS), which are generated by two arbitrary wave generators (AWG) with different orders. Here, in order to ensure the independence between two PRBS sources, 6 and 9 bit patterns with 4 Gbps data rate are adopted respectively. Considering the frequency range of SMA connector and dominant mode in SIW, the local oscillator with $20 \mathrm{GHz}$ signal carrier is chosen for modulation and demodulation. Since there are approximate $6 \mathrm{~dB}$ conversion losses caused by ever mixing, an amplifier is applied to improve signal amplitude in SIW. Two same lowpass filters with $7.5 \mathrm{GHz}$ cut-off frequency are also incorporated in the demodulation section to recover baseband signals.

The time domain waves of 4 Gbps PRBS in channel I and Q are shown in Fig. 6 (a), which are sampled by $80 \mathrm{GHz}$ digital signal analyzer (DSA). The corresponding measured output eye diagrams are presented in Fig. 6 (b). Moreover, it can be seen from Fig. 6 (b) that the eye height in channel Q is slightly less than the one in channel I. This is because the carrier is attenuated by two phase shifter when it reaches to the LO end of the mixer in channel Q. Furthermore, PRBS signal sources are composed by two AWG with different parameters such as rising/falling time and signal ripple. These differences mentioned above will affect the quality of eye pattern directly.

From the measurement of Vpp, the voltage lever greater than $120 \mathrm{mV}$ can fully assure a good eye opening. Its good performance has been experimentally demonstrated. The improved system is very capable of communication with 8 Gbps QPSK signals with excellent transmission quality. If we realize the same transmission rate by using of mixing scheme, two SIW interconnects are needed with 4 Gbps every channel, which will be at the cost of additional areas. However, the output eye opening begins to close when the data rate continues to increase for mixing scheme. We can conclude that it is not possible to demonstrate the system performance for much higher bit rates with the existing system components unless SIW with larger bandwidth is employed. 


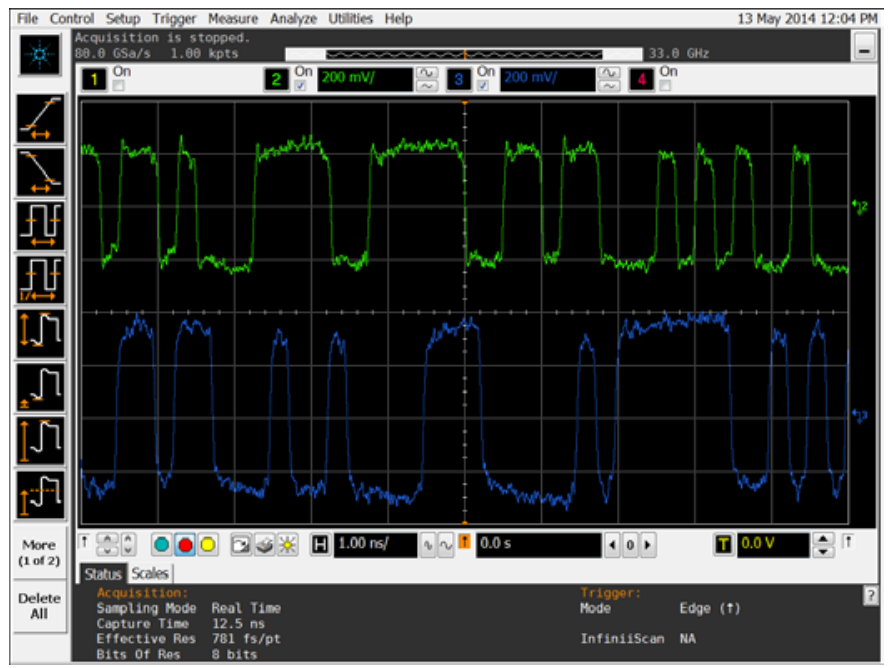

(a)

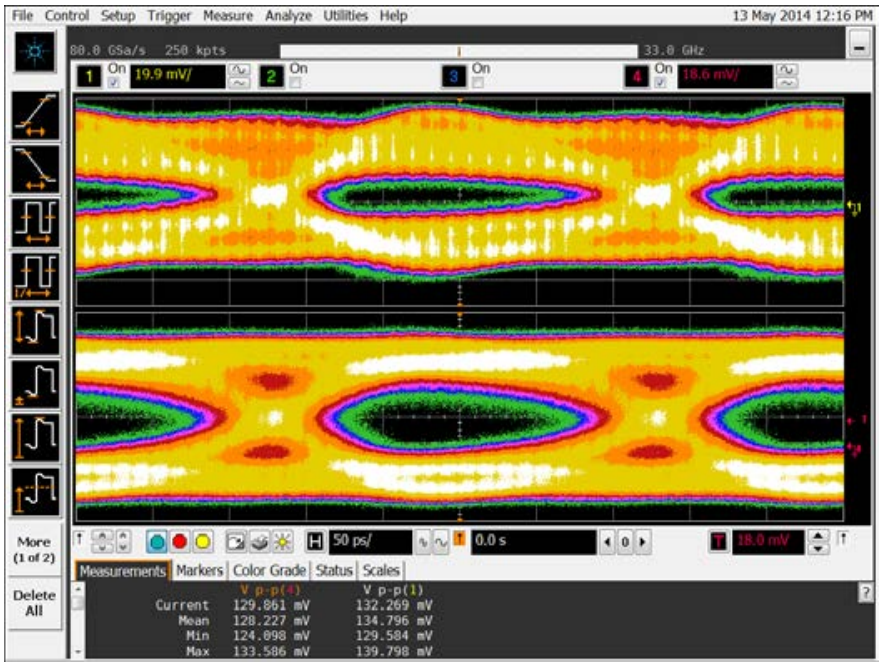

(b)

Fig. 6. Experimental results in channel I/Q (a) time domain waveforms (b) eye diagrams.

\section{Conclusion}

In this paper, SIW for operation in $14 \mathrm{GHz}$ bandwidth is designed and fabricated. A high speed data transmission system based on SIW employing QPSK is demonstrated in detail. Experimental results shows that the SIW system can achieve up to 8 Gbps data rate by using of QPSK technology. Overall, SIW interconnect has been proven to be an effect medium for delivering high-speed data transmission.

\section{Acknowledgement}

This work was supported by the Shanghai Science and Technology Committee under Grant 13511500900, Specialized Research Fund for the Doctoral Program of Higher Education under Grant 20120073130003, Director Fund of Key Laboratory of Ministry of Education of Design and Electromagnetic Compatibility of High Speed Electronic System under Grant 201401, and National Nature Science Foundation of China (No. 61234001 and No. 61327803). 


\section{References}

[1] A. Doghri, T. Djerafi, A, Ghiotto, and K. Wu, "Substrate Integrated Waveguide Directional Couplersfor Compact Three-Dimensional Integrated Circuits” IEEE Trans. Microwave Theory Tech., vol. 63, no. 1, pp. 209-221, Jan. 2015.

[2] H. D. Chen, W. Q Che, Q. Q. He, W. J. Feng, X. Wei, and K. Wu, "Compact Substrate Integrated Waveguide (SIW) Monopulse Network for $\mathrm{Ku}$-Band Tracking System Applications” IEEE Trans. Microwave Theory Tech., vol. 62, no. 3, pp. 472-480, Mar. 2014.

[3] Z. B. Wang, and C. W. Park, "Novel Substrate Integrated Waveguide (SIW) Type High Power Amplifier Using Microstrip-to-SIW Transition,” Asia Pacif Microwave Conf Proc APMC, pp. 101-103, Korea, November 2013.

[4] A. Suntives, and R. Abhari, "Ultra-High-Speed Multichannel Data Transmission Using Hybrid Substrate Integrated Waveguides," IEEE Trans. Microwave Theory Tech., vol. 56, no. 8, pp. 1973-1984, Aug. 2008.

[5] N. Sarkhosh, H. Emami, and M. Ashourian, "Carrier Frequency Independent RF Photonic QPSK Modulator,” IEEE Trans. Microwave Theory \& Tech., vol. 62, no. 2, pp. 373-379, Febuary 2014. 